8. OEOBQE'S HOSPITAI.

OLCRRATION OF THE LARGE INTESTINE, IN CONNEXION WITH STRANGULATED HERNIA. ILLUSTRATED BY SIX CASES.

(Communicated by T. Hor.ures, EsQ., Surgical-Registrar to the Hospital.)

I UAID before the readers of this JoURALI a short time ago the particulars of a case of ulceration of the large intestine, leading to effusion of fæces into the subcutaneous cellular tissue, and diffuse inflammation. In my present communication, I propose to pursue the subject of ulceration of the intestine, in connexion with strangulated hernia, by adducing some cases in which the gut contained in the hernial sac has been found after death to have been ulcerated, usually as a consequence of the pressure of the stricture, but sometimes (vide Case VI) apparently independent of such pressure, and even (Case I) without the presence of any stricture whatever. This latter, therefore, is a strictly parallel case to the one which I before reported, and the occurrence of hernia at the same time may be regarded as accidental, there being no proof that the sac (which was found empty at the time of operation) had ever contained intestine; or if so, that that intestine was in any way injured either by the parts around the sac or by the efforts at reduction. The six cases which I have brought together all occurred within two years, out of a series of thirty-eight operated on during that time at St. George's Hospital; and although differing in minor features, they all agree in the main one; viz., that the gut, which presented no sign of mortification at the time of operation, was found either thin, or after death to be perforated by ulceration, and that this condition was not indicated by any of the general symptoms usually recognised as attending gangrene of the intestine, and that the ulcerative action appeared to be, in many of the cases, unchecked by the divisions of the stricture (vide Cases III and rv), and was not in all cases connected either with long continued strangulation, or any injudicious manipulation. Instances of this kind must, I think, be taken into consideration in determining the question of opening or not opening the sac, as a general rule, in the operation for hernia, on which subject I would refer to an admirable article by Mr. Nathaniel Ward in a recent number of the Lancet. It would be very foolish in me to offer a confident opinion on a point on which so many men of great experience and matured powers of observation have come to opposite conclusions; but surely the fact that ulceration of the gut may exist in so many as one-sixth of a tolerably extensive series of cases, in which the strangulation has not been protracted, nor the symptoms very severe, is one which (if well established) cannot be without its weight in a question of this kind. The anatomy of this lesion I believe to be that the mucous membrane of the intestine becomes irritated, either from the pressure of the stricture, the irritation of some foreign body contained in the gut, or external violence; and that this action is accompanied by local inflammation on the serous surface of the neighbouring coils of intestine, by which adhesions are formed, blocking off the fæcal discharge, which follows the extension of the ulcerations, from the peritoneal cavity, and thus converting the case into an ordinary one of fæcal fistula. The prognosis then depends, in a great measure, on the patient's powers of constitution, as will be seen from the results of the appended cases.

CAsE I. Femoral Hernia: Exploratory Operation: Hernial Sac found empty: Artificial Anus in consequence of Ulceration of the Intestine: Death from exhaustion by bedsores. S. C., female, aged 46 years, was admitted April 5th, 1853. She had had femoral hernia on the left side for about three years, but had never worn a truss or taken any notice of it. Latterly she had been labouring under bronchitis; and after a fit of coughing, two days before admission, she noticed that the tumour was down. The bowels had been open on the previous day, on which day she had also had a fit of romiting. A medical man was sent for, who reduced the hernia, and the bowels acted on the following day. Soon afterwards the tumour reappeared; the medical attendant was again sent for, but could not reduce it.

She accordingly came to the hospital; warm bath and the taxis were applied, but the tumour, though diminished in size, could not be made to disappear.

Under these circumstances, it appeared right to perform an exploratory operation.

After division of the superficial parts, an enlarged gland was found, and behind this a loose flaccid membrane, which looked at first sight like a knuckle of intestine, but, on further examination, turned out to be the empty hernial sac. It contained only some serous fluid. The operator passed his finger up through its neck, which was constricted, into the peritoneal cavity. There were no alarming symptoms after the operation; the bowels were rather costive, but acted with purgatires. The pulse was weak and quick, and the tongue rather dry and brown; but this had been the case before the operation.

On the fifth day the bowels were observed to be confined; the belly swollen and tympanitic; a purge was given, and a natural evacuation obtained. In the evening, however, it was observed that fæcal matter exuded from the wound, and the next day all the fæces were passed by this artificial anus. Her weakness, and the low fever under which she was suffering, increased. She complained of a gond deal of tenderness around the wound, and her cough annoyed her considerably, so that it was feared that she would sink. Good diet and quinine, however, enabled her to rally, and she survived for about four weeks, during which fæces continued to pass by the wound, but in decreasing quantities, and occasionally for a short time wholly per anum. Her death appeared due entirely to the exhaustion of large bed-sores.

On post mortem examination, the convolutions of the small intestine were found matted together in many places by bands of adhesion of old standing; and several of the spaces left between the attachments of these adhesions were filled with pus, forming numerous circumscribed abscesses.

An aperture, sufficient to admit a large probe, remained in the left groin. On the inner surface of the abdominal wall, opposite to this point, a portion of the small intestine, about three feet from the ileo-crcal valve, was found to be closely adherent. The aperture above described communicated with this portion of the intestine, which was considerably dilated. This dilatation was continuous with the upper portion of the gut by a broad channel, and with the lower portion by a much narrower one, the constricted portion of the intestine just corresponding to the fistulous orifice. Below this orifice, and between it and the lower portion of the intestine, the gut presented a slightly dilated point; while, above the constriction, its whole calibre was to some extent dilated.

Case II. Femoral Hernia: Operation: Ulceration and Mortification of the Intestine: Death. G. P., male, aged 63, was admitted October 7 th, 1853 . This old man had had a femoral hernia for many years: it had been strangulated for about three days; and, on his admission, he was in so alarming a state of prostration that it was not thought proper to attempt reduction, or eren administer chloroform. There was no extraordinary difficulty about the operation, though the stricture was a tight one. The gut was found very dark in colour, but with no lymph on its surface ; and as it seemed not so far disorganised as to preclude the hope of its recovering itself, it was returned into the belly. Next day he had had several natural motions, and his pulse had rallied considerably; but there were slight symptoms of peritonitis. On inspecting this wound, a discharge was seen to proceed from it not unlike fluid fæces, though its character was not very certainly marked. When wiped away, it curled up in good quantity from deep in the wound. He sank rapidly from this time, the pulse becoming suddenly weaker, and at length quite imperceptible and he died in the night. 
On past morters examination, a large quantity of highly fwil fluid, of a yellow tinge, was found in the peritoneal crity. The peritoneal surface of the intestines was vascolar throughout, and their convolutions matted together. The portion of intestine that had been strangulated was found to be situated about two feet from the ileo-creal Valve; the constriction had not entirely surrounded the gut. The part which had been included in the hernia looked gangrenous, this appearance being most distinct in the ring marked by the stricture, where the gut had given way at one point.

Case IIr. Femoral Hernia: Operation: Clceration of the Intestines: no Effusion of Faces into the Peritoneal Cuvity: Death. Female, aged 76; March 27th, 1854. This old woman was admitted on account of strangulated femoral hernia. She had never observed any tumour in the groin till two days before admission; when it made its appearance, she was straining at stool. The bowels had not acted since that period, and romiting had come un, but not immediately. On her admission, there was seen a largish tumour lying over Poupart's ligament, not tender, nor inflamed externally. There was tympanitic, but no great pain in the stomach, and no general symptoms. Taxis was applied unsuccessfully; then ice to the tumour for about six hours; and then, after a second application of the taxis, the operation was performed.

The bulk of the tumour was found to be due to a large accumulation of fat, the hernia itself being extremely sall. The sac was opened, and contained only a small bonckle of intestine, much inflamed, and coated with a thick layer of lymph, but showing no signs of gangrene. She was much relieved by the operation, and went on well till the next day at noon, when the vomiting rocurred, the matters ejected being evidently stercoraccous. This vomiting went on during the rest of the case, accompanied at first by obscure symptoms of low peritonitis. She secmed to be benefited by the administration of solid opium, and, when the tympanitis and tenderness of the belly had disappeared, by wine and brandy. She continued to sink, however, and dicd five days after the operation. On the day of her death, for the first time (though this point had been particularly attended to), a fæcal odour was observed to proceed from the wound. The bowels had not acted since the operation.

On post mortem examination, a very few shreds of recent fibrin were found on one or two of the convolutions of the intestines. On removing a knuckle of the small intestine from the right inguinal region, it was found to have been slightly adherent to the anterior wall of the belly, and to a subjacent portion of the ileum. This latter was the portion that had been strangulated, and at its margin was an ulcerated opening, filled with tolerably solid frecal matter, which was prevented from escaping into the peritoneal cavity by the adhesion of the piece of intestine before mentioned.

Case Iv. Femoral Ilernia strangulated two Days: Facal Pistula seven Days after the Operation: Recovery. S. D., female, aged 46, had had femoral hernia for about eight years, but had never given any attention to it, nor suffered any inconvenience from it, till the morning before her admission, when the usual symptoms of strangulation occurred. The taxis was applied on the morning of her admission, but not violently. When admitted, she was in a atate of extreme prostration, so that it was almost feared that she would die unrelieved. By the application of stimulants, however, she was so far restored as to be able to undergo the operation, which was performed about thirty-six hours after strangulation. On opening the sac, a piece of small intestine was found in it, very dark coloured, and coated with lymph. The sac contained also some serous fluid. She went on well for the first week; but on the 7 th day frecal discharge occurred from the wound, and continued for about a fortnight. She was in an alarming state of weakness for several days, with livid extremities, and cold clammy face; but recovered gradually, and was discharged cured in about four weeks from the operation.
Case v. Femoral Hernia: Stranguleted two days : Small Perforation found in the Gut at the Operation: Ligature pieced around this Penfontion: Recovery. I. Y., fomale, aged 46, had had strangulated femoral hernia for about two days, and was in a stato of great distress when admitted into the hospital, with hard and frognent puloe, tympanitic, pain in the belly, foul tongue, and very anxious expression. The hernial tumour also was very tender and red. As attempts at reduction had been made before her admigsion, the operation was at once proceeded with.

On the sac being exposed, and a small incision carefully made into it, a quantity of fluid frecs escaped. The incision was then enlarged, and the state of the gut examined. The latter was found distended, very dark in colour, and a small ecchymosed spot, about as large as a fourpenny-piece, was seen on its surface. The stricture, which was very tight, was now incised. On the gut being manipulated, its contents (fæcal fluid mixed, as it appeared, with pus) escaped, and the intestine collapsed. On minute examination, a small perforation, about as large as a pin-hole, was found in the centre of the ecchymosed spot above noticed. This was surrounded with a fine ligature, the ends of which were cut close, and the gut returned into the abdomen.

She recovered, with no bad symptoms, except an attack of phagedæna of the external wound.

CASE VI. Oblique Inguinal Hernia: Strangulation (slight) one day: Few General Symptoms: Ulceration of the Gut, and Frecal Discharge on the eighth day: Death. G. S., male, aged 58 , had had scrotal hernia for a very long time, which, according to his story, had always been partially reducible till three days before his admission, when the bulk of the tumour had been observed to be increased, and the hernis to be entirely irreducible. He had suffered from vomiting during these three days, and from constipation during the day of his admission ; the romit had not, as far as was knorn, been of a fæcal character. The hornia was very red and tender, but not particularly tense. On bandling it, a gurgling sensation was perceived; but no impression was made on the bulk of the tumour. The general symptoms were slight.

Attempts at taxis were made, both without and under the influence of chloroform; and these proving ineffectual, it was resolved to expose the contents of the sac. The latter was covered by numerous layers of condensed cellular tissue ; on its being laid open, a large piece of the great intestine was found in it, covered with its appendices epiploicæ matted together, which were connected by soft recent adhesions to the front of the sac, while the gut itself was firmly united to its posterior layer by an old false membrane, which it was impossible to separate. The constriction was very trifling; so that, after the adhesions in front had been broken down, the finger passed readily into the cavity of the belly. The gut was necessarily left unreduced.

I must not dwell at length upon the symptoms of this remarkable case: suffice it to say, that for the first week he did not present a bad symptom, except (what I think I have uniformly observed in these cases) extreme tympanitis; that on the eighth day reces came through the wound in small quantities, and in much larger quantity on the day following, without his displaying any symptom of constitutional disturbance ; that this frecal dischargo (which used to intermit occasionally, and never appeared to exhaust the whole contents of the bowels) had in tho course of a month so nearly ceased that the man was able to move about the ward; and that one day while he was doing so the adhesions of the wound gave way, and a gush of fres showed that the free communication of the intestiue with the air was re-established; that the mucons membrane of the gat then became prolapsed at this artifcial anus; and that an instrument, applied with a view of supporting this prolapsus and retaining the contents of the bowel, unfortunately produced sloughing, which assumed the phagedxnic character; and that, exhausted by this sloughing, and worn out by the horrible consequences of an immense opening into the large intestine at the groin (for the sloughing had greatly enlarged the artificial anies 
at wall as the external wound), he gradually mant, and died chout three months after the operation.

Post mortem examination showed the iloum and colon, terminating seperately in the wound, the mucous membrane of the intestine being continuous with the rest of the wound, co as to shut off the cavity of the gut from that of the poritoneum.

\section{OHiginal Communitations.}

\section{CASES IN MIDWIFERY.}

By Thomas RapFond, M.D., F.R.C.P.Edin., etc., Consulting Physician to St. Mary's Hospital, Manchester.

\section{SECOND SERIES.}

THIs series of cases will include those which are accompanied with hæmorrhage-commencing with those of "placenta prævia".

CaSE I. On June 24th, 1820, I was requested by Mr. Dick to visit a woman who resided in Greengate, Salford, who was reported to be flooding: she was in labour of her third child, and had a profuse discharge of blood. I found she had previously fooded twice, at intervals of about three weeks; but each of these attacks was comparatively trifling. There was no abvious cause for them; and on the patient being put to bed, the discharge soon began to diminish, and required no further measures besides rest in the recumbent position.

The present flow had abated when I arrived; but it was, and had been, increased on the accession of pain. On making an examination, I found the os uteri dilated to about the size of a crown-piece; it was soft and dilatable; a small partion of the placenta with the membranes was felt tightened during the pain, but hanging loosely over the os when it (the pain) subsided. The head of the child was easily touched, as the uterus acted briskly and effectively; and as the discharge of blood was now comparatively trifling, it was thought prudent not to interfere, but to leave the case to nature. In the course of two hours, the membranes ruptured; the head descended into the pelvis, pressing the separated portion of the placenta aside as it progressed, and very soon the child was born alive. The uterus contracted well; there was only an ordinary discharge; in about half an hour afterwards, the placenta was expelled. No further hemorrhage happenod. The bandage placed on before delivery was now tightened. She recovered without the slightest interruption. Those portions of the placenta which had been separated previous to labour, were readily distinguished from that (the last) which caused the flooding at this time, being more consolidated and of a different colour.

Case II. Mrs. Buckley (on 24th March 1821) desired me to visit a hospital patient residing in Hanover Street, who was said to be in labour, and flooding. When I arrived, I found the discharge, which at first was great, considerably abated. This was her fifth pregnancy. She had felt pains four hours, which had now become severe during each pain the flow of blood had been slightly increased from the commencement of the labour, but was trifling in amount until the time when $I$ was sent for. Her pulse was, however, unaffected, and her countenance natural. The os uteri was dilated to the size of a halfcrown, and felt thin and soft; throngh it, I found an edge of the placenta, which organ adhered to the right lateroposterior part of the cervix uteri; the distended membranes were readily perceived during the pain, and the head of the child was felt on its remission.

I remained with the patient some time, during which the prins increased in intensity and effect; the discharge of blood gradually cessed. I now left her in the charge of the midwife, with an express order to send for me agin if the bleoding recurned I heard nothing until the following day, when she called, and stated the membranes ruptaned in about two hours after I left; the child was born alive; the placenta was naturally expelled; there was no further loss than what usually takes place. The woman reoorered well.

CasE III. On Febreary 21st, 1822, I was requested by Mrs. Bradley to visit a hospital patient residing in Cumberland Street, who was reported to be flooding. She had now arrived at the eighth month of her socond pregnancy, and had been quite well up to this period. She began flood without any known cause. Mrs. Bradley had put her to bed, and placed a bandage round her, and used cold applicatious. The flow of blood was said to have been profuse, but it had considerably lessened when I saw her. I examined her, and found the os uteri closed, and therofore I could not ascertain whether the placenta was situated noar it or not, although I suspected it was, from foeling the cervical walls fuller and thicker than they ordinarily are at this period of pregnancy; and also, as no external cause had occurred to acoount for the accidant.

When I left the patient, I desired Mrs. Bradley to send again for me if there was a return of the hemorrhage. I however heard nothing more of the case until a month had elapsed, when I was called to her to extract the placenta, which I found slightly adherent. Her labour up to this point had been natural and easy. The placenta was readily detached and remored, and there was no flooding. I caro fully examined this organ, and readily distinguished thest portion which had been separated and caused the accilent from the rest of it; its structure had become firmly condensed, and less vascular.

CASE Iv. In June 1830, I was requested to visit a lady pregnant for the third time. My opinion was sought, bo cause in her two former labours the children were been dead. She was now nearly seven months advanced; her health was good; and she said she felt the child very lively. In a fortnight after this interview, I was sent for, as she was flooding. I found her on the bed, and looking pale; her pulse was rather quick and foeble. I was told she had lost a large quantity of blood. Although the bleeding was now comparatively trifling, yet it continued. She had no pain; and having placed my hand on the abdomen, I felt the uterus flaocid; I found the os utcri closed and the cervix uneffaced. A bandage was placed round the body, and pinned tightly over the uterus. Cold water with rincgar was externally applied, and the apartment kept cool. An unstimulating diet was ordered, and quietude in the recumbent position strictly enjoined. Nothing further was required, as the hromorrhage gradually ceased, and did not return. She progressively improved after this accident, and she continued to feel the child to be vigorously alive. Arrived at the eighth month, it wras for a few days felt less sensibly, and then altogether ceased to move. At this time the breast lessened in size, and became mere flaccid. About a week before the expiration of the ninth month, labour came on. When I visited her, the pains, now brisk and frequent, had existed for two hours. I found the os uteri consideribly dilated, soft, and yielding. The membranous bag, with a portion of the placenta, felt ; but there was not the slightest discharge of blood. The os uteri continued rapidly to dilate; the membramen ruptured; and the separated (now loose) flap of the plo centa was pushed aside, and passed by the descending head. The pains being very strong and forcing, the child (dead) was soon expelled. There was not the slightest hrmorrhage. The placenta was soon extruded, which, on examination, was found had lost its usual fresh vasoutar character; the portion which had been separated at nearly the seventh month, and then caused the flogding, readily fixed upon.

CasE ₹. The following cese recently occurred to my respected friend and colleague Mr. Skinner, who has kindly permitted me to cite it :-

On October 28th, 1855, 5 o'clock P.r., I was called by one of the midwires to attead Ann Maton, aged 23 years who had been attacked, while in bed this morning, with 